\title{
CORRECTION
}

S. M. Abo-Dahab

\section{Correction to: A two-temperature generalized magneto- thermoelastic formulation for a rotating medium with thermal shock under hydrostatic initial stress}

Received: 12 October 2020 / Accepted: 14 October 2020 / Published online: 3 November 2020

(C) Springer-Verlag GmbH Germany, part of Springer Nature 2020

Correction To: Continuum Mech. Thermodyn. (2020) 32:883-900

https://doi.org/10.1007/s00161-019-00765-3

Unfortunately, the original version of the article contained error in the below equation terms. The correct equation terms should read as below

In Eq. (12), the term $-\gamma T_{0} \frac{\partial T}{\partial x}$ must be substituted with $-\gamma \frac{\partial \theta}{\partial x}$

In Eq. (13), the term $-\gamma T_{0} \frac{\partial T}{\partial y}$ must be substituted with $-\gamma \frac{\partial \theta}{\partial y}$

In Eq. (17), the term $-\frac{\gamma}{\rho C_{0}^{2}} \frac{\partial \theta}{\partial x}$ must be substituted with $-\frac{\gamma T_{0}}{\rho C_{0}^{2}} \frac{\partial \theta}{\partial x}$

In Eq. (18), the term $-\frac{\gamma}{\rho C_{0}^{2}} \frac{\partial \theta}{\partial y}$ must be substituted with $-\frac{\gamma T_{0}}{\rho C_{0}^{2}} \frac{\partial \theta}{\partial y}$

The right form of Eq. (A.5) is $h^{\prime}=\frac{\mu_{e} H_{0}}{2 \mu+\lambda} h$

In Tab. 1, the right dimensions of $\eta$ are $s \mathrm{~m}^{-2}$ and the right dimensions of $\rho$ are $\mathrm{kg} \mathrm{m}^{-3}$

Publisher's Note Springer Nature remains neutral with regard to jurisdictional claims in published maps and institutional affiliations.

The original article can be found online at https://doi.org/10.1007/s00161-019-00765-3.

S. M. Abo-Dahab ( $\varangle)$

Mathematics Department, Faculty of Science, Taif University, Taif 888, Saudi Arabia

E-mail:sdahb@yahoo.com 